\title{
机器学习方法用于建立乙酰胆碱酯酶抑制剂的分类模型
}

\author{
杨国兵 ${ }^{2}$ 李泽荣 ${ }^{1, *}$ 饶含兵 ${ }^{1} \quad$ 李象远 $^{2} \quad$ 陈宇综 ${ }^{3}$ \\ ('四川大学化学学院, 成都 610064; ${ }^{2}$ 四川大学化学工程学院, 成都 610065; \\ ${ }^{3}$ Department of Pharmacy, National University of Singapore, Singapore 117543)
}

\begin{abstract}
摘要: 我们构建了表征乙酰胆碱酥酶抑制剂分子组成、电荷、拓扑、几何结构及物理化学性质等特征的 1559 个描述符, 通过 Fischer Score 排序过滤和 Monte Carlo 模拟退火法相结合进行变量篎选得到 37 个描述 符, 然后分别用支持向量学习机(SVM)、人工神经网络(ANN)和 $k$-近邻 $(k-N N)$ 等机器学习方法建立了乙酰胆碱 酯酶抑制剂的分类预测模型. 对于训练集的 515 个样本, 通过五重交叉验证, 各机器学习方法对正样本, 负样本 和总样本的平均预测精度分别为 $87.3 \%-92.7 \%, 67.0 \%-81.0 \%$ 和 $79.4 \%-88.2 \%$; 通过 $y$-scrambling 方法验证 SVM 模型是否偶然相关, 结果正样本, 负样本和总样本的平均预测精度分别为 $72.7 \%-82.5 \%, 41.0 \%-53.0 \%$ 和 $62.1 \%-69.1 \%$, 明显低于实际所建模型的预测精度, 表明所建模型不存在偶然相关; 对 172 个没有参与建模 的外部独立测试样本, 各机器学习方法对正样本, 负样本和总样本的预测精度分别为 $93.3 \%-100.0 \%$, 74.6\%-89.6\%和 86.1\%-95.9\%. 所建模型中, SVM 模型预测精度最好, 且明显高于其它文献报道结果.
\end{abstract}

关键词: 乙酰胆碱酯酶抑制剂; 机器学习方法; 变量筛选; 应用域 中图分类号: 0641

\section{Classification Models for Acetylcholinesterase Inhibitors Based on Machine Learning Methods}

\author{
YANG Guo-Bing ${ }^{2} \quad$ LI Ze-Rong ${ }^{1, *} \quad$ RAO Han-Bing ${ }^{1} \quad$ LI Xiang-Yuan ${ }^{2}$ CHEN Yu-Zong ${ }^{3}$ \\ ( ${ }^{1}$ College of Chemistry, Sichuan University, Chengdu 610064, P. R. China; ${ }^{2}$ College of Chemical Engineering, Sichuan \\ University, Chengdu 610065, P. R. China; ${ }^{3}$ Department of Pharmacy, National University of Singapore, Singapore 117543)
}

\begin{abstract}
A total of 1559 molecular descriptors including constitutional, charge distribution, topological, geometrical, and physicochemical descriptors were calculated to encode acetylcholinesterase inhibitors. The 37 molecular descriptors were selected using a hybrid filter/wrapper approach by combining a Fischer Score and Monte Carlo simulated annealing. Classification models for the acetylcholinesterase inhibitors were then built based on support vector machine (SVM), artificial neural networks (ANN), and $k$-nearest neighbor $(k-N N)$ methods. For the 515 samples in the training set, we obtained average prediction accuracies of $87.3 \%-92.7 \%, 67.0 \%-81.0 \%$, and $79.4 \%-88.2 \%$ for the positive, the negative, and the total samples, respectively, by 5 -fold cross validation. Average prediction accuracies of $72.7 \%-82.5 \%$, $41.0 \%-53.0 \%$, and $62.1 \%-69.1 \%$ were obtained for the positive, the negative, and the total samples, respectively, by the $y$-scrambling method, indicating that there was no chance correlation in our models. An external test was conducted on 172 samples that were not used for model building and we obtained prediction accuracies of $93.3 \%-100.0 \%, 74.6 \%-89.6 \%$, and $86.1 \%-95.9 \%$ for the positive, the negative, and the total samples, respectively. The prediction accuracies obtained by all the machine learning methods especially by the SVM method were far better than previously reported results.
\end{abstract}

Received: July 22, 2010; Revised: August 15, 2010; Published on Web: October 11, 2010.

"Corresponding author. Email: lizerong@scu.edu.cn; Tel: +86-28-85406139.

The project was supported by the National Natural Science Foundation of China (20973118).

国家自然科学基金(20973118)资助项目

(C) Editorial office of Acta Physico-Chimica Sinica 
Key Words: Acetylcholinesterase inhibitor; Machine learning method; Feature selection; Applicability domain

阿尔茨海默病(Alzheimer disease, AD)即老年性 痴呆症, 是慢性进行性神经退化疾病, 严重影响患 者的认知功能、记忆功能、个人生活能力和情感人格 等, 65 岁以上人群中大约 $10 \%$ 患有此病. 一直以来, 世界上较为接受的 $\mathrm{AD}$ 病理为 “胆碱能缺失学说”, 学说认为老年性痴呆症患者大脑内神经递质乙酰胆碱的缺失是导致 $\mathrm{AD}$ 疾病的关键原因 ${ }^{[1-2]}$. 乙 酰胆碱酯酶(AChE)会催化乙酰胆碱的裂解反应, 导 致乙酰胆碱的缺失、神经信号传递失败. 目前对老 年痴呆的药物治疗主要是通过用乙酰胆碱酯酶抑 制剂(AChEIs)来减少乙酰胆碱的消耗, 从而提高胆 碱能神经元的兴奋性, 达到治疗的效果 ${ }^{[3]}$. 所以乙酰 胆碱酯酶抑制剂作为主要治疗手段已经被广泛研 究. 1993 年, 第一个乙酰胆碱酯酶抑制剂他克林(tacrine)用于临床治疗 $\mathrm{AD}^{[4]}$, 此后, 越来越多的 $\mathrm{AChEIs}$ 被开发出来, 比如多奈哌齐(donepezil) ${ }^{[5]}$, 加兰他敏 (galantamine) $^{[6]}$, 利凡斯的明(rivastigmine) $)^{[7]}$.

事实上, 实验方法开发 AChEIs 是比较盲目的, 因此用计算的方法在实验之前先进行针对性的篎 选 AChEIs 是必要的, 可以为实验提供理论指导, 挑 选出一些有用的分子供实验分析, 这样减少了药物 开发的盲目性, 大大节省了开发的时间和资金. 到 目前为止, 人们已经开发出了许多计算方法或模型 去预测 AChEIs, 比如定量构效关系 $(\mathrm{QSAR})^{[8-11]}$, 分 子对接(docking) ${ }^{[12-14]}$. 最近, Lv 等 ${ }^{\left[{ }^{[15}\right]}$ 把三种机器学习 方法 SVM, $k-\mathrm{NN}$ 和决策树(DT)用于建立 AChEIs 分 类模型, 对抑制剂的预测精度在 $76.3 \%-88.0 \%$, 非 抑制剂的预测精度在 $74.3 \%-79.6 \%$, 但是他们用的 数据不够多, 预测结果也不够理想. 为此, 本文着力 于用机器学习方法 $\left(\mathrm{SVM}^{[16]}, \mathrm{ANN}^{[17]}, k-\mathrm{NN}^{[18]}\right)$ 使用更 多的样本去开发预测精度更高的模型, 为篮选出有 用的 AChEIs 提供更有效的手段.

\section{1 原理与方法}

\section{1 数据收集}

本文收集的 717 个抑制剂化合物分子(抑制剂 的基本结构单元见 Scheme 1)及它们的实验半数抑 制浓度 $\mathrm{IC}_{50}$ 主要来源于参考文献[12,19-55]. 同文献 [15]一样, 我们将 $\mathrm{IC}_{50} \leq 400 \mathrm{nmol} \cdot \mathrm{L}^{-1}$ 的化合物划分为 抑制剂(正样本), 将 $\mathrm{IC}_{50} \geq 600 \mathrm{nmol} \cdot \mathrm{L}^{-1}$ 的化合物划分
为非抑制剂(负样本). 这样, 717 个分子包含了 420 个抑制剂和 267 个非抑制剂, 此外, 还有 30 个化合 物的 $\mathrm{IC}_{50}$ 在 400 和 $600 \mathrm{nmol} \cdot \mathrm{L}^{-1}$ 之间, 和文献 [15]一 样, 这里也不把他们归入抑制剂或非抑制剂, 不对 它们进行研究. 本文所研究分子的稳定几何结构均 用 HyperChem 7.0(http://www.hyper.com/)中的 MM+ 力场优化得到.

\section{2 数据集的结构多样性}

数据集中化合物的结构多样性可以通过多样 性指标(DI)来评定, DI 值是指数据集中成对化合物 间的不相似性 ${ }^{[56]}$ :

$$
\mathrm{DI}=\frac{\sum_{i=1}^{N} \sum_{j=1, i \neq j}^{N} \operatorname{diss}(i, j)}{N(N-1)}
$$

其中 $N$ 是数据集中化合物的个数, $\operatorname{diss}(i, j)$ 是化合物 $i$ 和 $j$ 之间不相似度的度量. 因为不相似度和相似度 是互补的, 所以经常定义为:

$$
\mathrm{DI}=1-\frac{\sum_{i=1}^{N} \sum_{j=1, i \neq j}^{N} \operatorname{sim}(i, j)}{N(N-1)}
$$

其中, $\operatorname{sim}(i, j)$ 是化合物 $i$ 和 $j$ 之间的相似度的度量, 本文中, 相似度采用常用的 Tanimoto 系数 ${ }^{[57]}$, 定义 如下:

$$
s(i, j)=\frac{\sum_{d=1}^{l} x_{d i} x_{d j}}{\sum_{d=1}^{l} x_{d i}^{2}+\sum_{d=1}^{l} x_{d j}^{2}-\sum_{d=1}^{l} x_{d i} x_{d j}}
$$
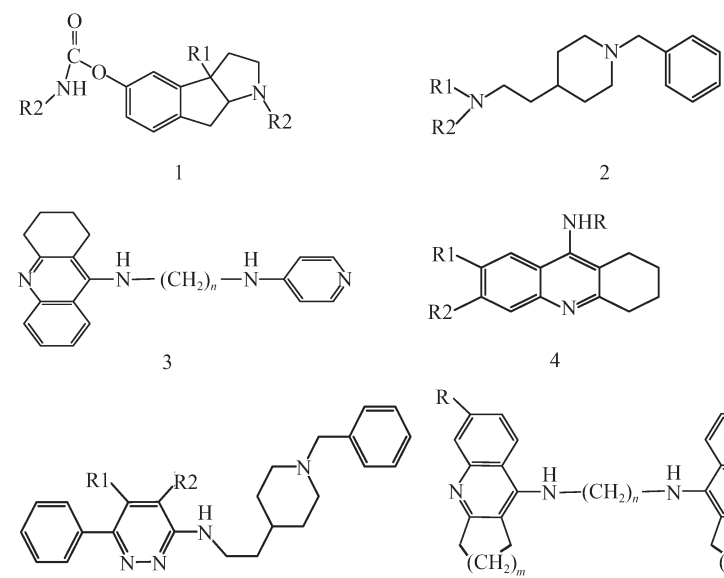

5
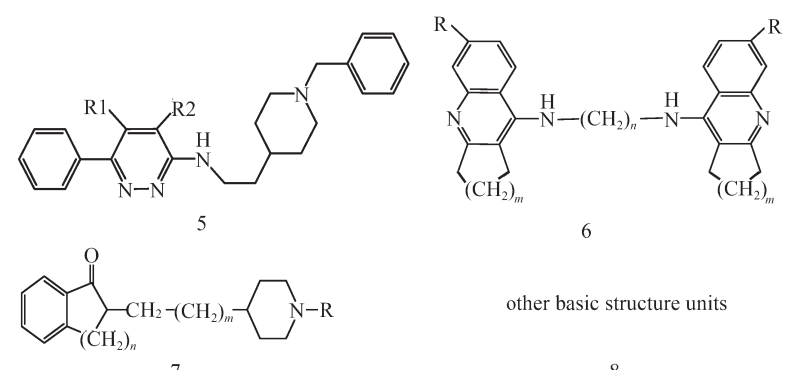

other basic structure units

Scheme 1 Basic structure units of acetylcholinesterase inhibitors 
其中 $l$ 是描述符的个数, $x_{d i}$ 和 $x_{d j}$ 分别是化合物 $i$ 和 $j$ 的第 $d$ 个描述符, $s(i, j)$ 为 Tanimoto 系数. 由计算可知 连续的 Tanimoto 系数的变化范围为 $(-0.333,1.0)$, 所以将 Tanimoto 系数归一化, 使其范围为 $(0,1.0)$. 归一化的过程通过下式实现:

$$
\operatorname{sim}(i, j)=\frac{s(i, j)+0.333}{1.333}
$$

这样代入式(2)得到的 DI 值的变化范围为 $(0,1.0)$, 数 据集化合物的结构多样性随着 DI 值的增大而增加.

本文研究的 687 个分子的 DI 值为 0.738 , 表明所 研究对象具有足够的结构多样性.

\section{3 分子描述符的计算及䇻选}

分子描述符是构建模型的关键, 本研究我们采 用 MODEL 软件 ${ }^{[58]}$ 进行描述符的计算, 此软件在网 址(http://jing.cz3.nus.edu.sg/cgi-bin/model/model.cgi) 可寻, 是免费的学术软件. 我们对每个分子计算了 1559 个描述符, 其中包含 20 个组成描述符、21 个电 荷相关描述符、2 个物理化学性质描述符、482 个拓 扑描述符和 1034 个几何描述符.

很显然, 并不是所有 1559 个描述符都与分子的 活性相关, 而且有些描述符之间存在线性相关, 所 以需要选择合适的描述符以使建立的模型达到最 好的预测能力. 本文采用一套混合描述符篎选方法 来寻找最优的描述符子集, 其步骤如下.

(1) 预处理: 首先, 如果有 $90 \%$ 样本的同一个描 述符值是相同的, 那么这个描述符对区别分子的活 性无效, 将它去除; 其次, 对任意一个描述符, 遍及 所有样本计算该描述符的相对标准偏差, 如果其值 小于 0.05 , 也将它删除; 最后, 如果两个描述符的 Pearson 相关系数 ${ }^{[59]}$ 大于 0.9 , 说明这两个描述符高 度相关, 删除其中之一.

(2) 描述符排序: 经过预处理后的描述符, 按照 Fischer Score $(F-\text { Score })^{[60]}$ 值递减的顺序排序. F-Score 值 $F(i)$ 是单个描述符的分辨能力的简单度量, 定义 为:

$$
F(i)=\frac{\left(\mu_{i}^{+}-\mu_{i}^{-}\right)^{2}}{\left(\sigma_{i}^{+}+\sigma_{i}^{-}\right)^{2}}
$$

其中 $\mu_{i}^{+}$和 $\mu_{i}^{-}$分别是第 $i$ 个描述符的正、负样本的平 均值, $\sigma_{i}^{+}$和 $\sigma_{i}^{-}$分别是它们的标准偏差. 描述符的 $F$ Score 值越大, 它对样本的区分能力就越强. 具体的 簰选步骤如下: (1) 计算每一个描述符的 F-Score 值, 并将所有描述符按照 $F$-Score 值从大到小的顺序排 列; (2) 把训练集用五重交叉验证的方法得到 SVM
模型的平均推广误差, 并调节模型参数 $\sigma$ 使推广误 差最小; (3) 删除排序靠后 5 个描述符, 回到第(2)步, 直至模型的推广误差最小. 由此, 可选择出 $F$-Score 值最好的描述符子集及对应的模型参数 $\sigma$.

(3) Monte Carlo 模拟退火算法: 描述符在经过 上述两个步骤过滤后, 进一步用 Monte Carlo 模拟退 火法结合 SVM 进行篮选, 文献[61-62]有类似描述. 首先我们给定一较高的模拟退火起始温度 $T_{0}$, 以及 每一温度的平衡步数和终止温度, 然后具体的篮选 步骤如下: (1) 给定 SVM 高斯指数的初值 $\sigma$; (2) 在初 始温度 $T_{0}$ 下随机选择一组描述符子集作为初始解; (3) 用五重交叉验证计算 SVM 模型的平均推广误差 $E_{\text {old }}$; (4) 对描述符子集给一随机微扰, 得到一新描述 符子集, 按上述方法计算模型的平均推广误差 $E_{\text {new }}$; (5) 如 $E_{\text {new }}<E_{\text {old }}$, 则接受新的描述符子集代替原来 的描述符子集. 反之, 则以概率 $P=\exp \left\{-\left(E_{\text {new }}-E_{\text {old }}\right) /\right.$ $T\}$ 接受为新描述符子集; (6) 回到步骤(4), 直到达到 此温度下的最大平衡步数; (7) 降低模拟退火温度 $T$, 重复上步温度的篎选过程, 直到达到指定的终止退 火温度; (8) 系统地调整 $\sigma$ 值, 返回步骤(2), 直至 SVM 模型的平均推广误差最小. 由此, 可得到最优的描 述符子集和对应的最佳模型参数 $\sigma$.

\section{4 数据集的划分和模型的验证}

本研究采用较流行的 $\mathrm{KS}$ 方法 ${ }^{[63]}$ 来设计训练集 和外部独立测试集. 所构成的训练集包含 315 个正 样本和 200 个负样本, 外部独立测试集包含了 105 个 正样本和 67 个负样本. 训练集用于模型的构建, 外 部独立测试集用于客观评价已建模型的预测能力.

本文中我们用三种方法去验证分类模型. 第一 种是五重交叉验证, 把训练集随机分为 5 个数量接 近的子集, 每次用其中 4 个作为训练集, 另外一个作 为测试集, 这样重复 5 次, 保证每个子集都用作测试 集一次. 在此文中, 我们用五重交叉验证去优化预 测模型: 选出最优的描述符子集, 优化模型参数如 $\mathrm{SVM}$ 中的高斯指数 $\sigma, \mathrm{ANN}$ 中隐含层的个数, $k-\mathrm{NN}$ 中的 $k$. 第二种验证方法是 $y$-scrambling, 用来评估所 建模型是否偶然相关. 保持训练集样本输入变量不 变, 随机将部分样本的分类标号打乱, 用打乱类别 的训练集建立新的分类模型, 新模型的预测能力应 该大大低于基于原始数据建立的实际模型 ${ }^{[64]}$, 否则 就是偶然相关, 偶然相关的模型没有预测能力. 第 三种验证方法是外部独立测试 ${ }^{[6]}$, 把训练集中所有 样本基于选出的最优描述符和最佳参数建立预测 
模型, 用外部独立测试集来评价已建模型的预测能 力. 因为外部独立测试集中的样本没有参与模型的 开发, 所以这种验证方法比交叉验证更为严谨, 更 能客观评价模型的预测能力.

\section{5 机器学习方法}

\subsubsection{SVM方法}

SVM 方法是基于结构风险最小化准则, 即通过 对推广误差风险最小化, 从而实现模型的最大推广 能力. 该方法在文献 ${ }^{[16]}$ 中有详细介绍, 这里我们只做 简述.

给定一训练集 $\left\{x_{i}, y_{i}\right\}, i=1,2, \cdots, n, y_{i} \in\{-1,+1\}$, 其中 $n$ 是训练集样本的个数, $y_{i} \in\{-1,+1\}$ 是类别标 号. 如果训练集是线性可分的, SVM 就是构建超平 面

$$
f(x)=\mathbf{w} \cdot x_{i}+b=0
$$

使正样本和负样本可分, 且使其边界上的点到该超 平面的距离最大, 这可转化为下面的条件

$$
\begin{aligned}
& \mathbf{w} \cdot x_{i}+b \geq+1, y_{i}=+1 \\
& \mathbf{w} \cdot x_{i}+b \leq-1, y_{i}=-1
\end{aligned}
$$

限制下求函数

$$
\phi(\mathbf{w}, b)=\frac{1}{2}\|\mathbf{w}\|^{2}=\frac{1}{2}(\mathbf{w} \cdot \mathbf{w})
$$

的最小值. 由 Lagrange 乘数法可得解

$$
\mathbf{W}^{*}=\sum_{i=1}^{n} \alpha_{i}^{*} y_{i} \mathbf{x}_{i}
$$

上式满足限制条件 $\sum_{i=1}^{n} \alpha_{i}^{*} y_{i}=0$, 并有最优分类决策函 数:

$$
f(\mathbf{x})=\operatorname{sgn}\left(\left(\mathbf{w}^{*} \cdot x\right)+b^{*}\right)=\operatorname{sgn}\left(\sum_{i=1}^{n} \alpha_{i}^{*} y_{i}\left(\mathbf{x}_{i}, x\right)+b^{*}\right)
$$

其中, $\operatorname{sgn}()$ 为符号函数. 如果训练集是非线性可分 的, 则将向 $x$ 通过非线性函数 $\phi(x)$ 投影到某个高维 特征空间, 使其线性可分. 非线性函数 $\phi(x)$ 的引入 是通过核函数 $\boldsymbol{K}\left(x_{i}, x\right)$ 来实现的, 即 $\boldsymbol{K}\left(x_{i}, x\right)=\left(\phi\left(x_{i}\right)\right.$. $\phi(x))$. 此时的分类判别函数变为:

$$
f(x)=\operatorname{sgn}\left(\sum_{i=1}^{n} \alpha_{i}^{*} y_{i} \boldsymbol{K}\left(x_{i}, x\right)+b^{*}\right)
$$

核函数 $\boldsymbol{K}\left(x_{i}, x\right)$ 可以有多种形式, 本文使用 Gaussian 核函数:

$$
\boldsymbol{K}\left(x_{i}, x\right)=\exp \left(-\left\|\left(x_{i}-x\right)\right\|^{2} / 2 \sigma^{2}\right)
$$

式中高斯指数 $\sigma$ 称为模型参数.

\section{5 .2 其他机器学习方法}

此外, 还分别将三层(包括输入层、隐含层和输 出层)反向传播 $\mathrm{ANN}^{[17]}$ 和 $k-\mathrm{NN}^{[18]}$ 方法用于建立乙酰 胆碱酯酶抑制剂的分类预测模型. 各机器学习方法 的模型参数, 即 SVM 中的高斯指数 $\sigma, \mathrm{ANN}$ 中隐含 层的个数, $k-\mathrm{NN}$ 中的 $k$, 均是通过五重交叉验证使模
型的推广误差最小来优化. 上述方法均采用本小组 自编的程序来实现.

\section{6 结果评价}

我们采用普遍使用的方法来评估分类模型对测 试集的预测能力 ${ }^{[6]}$ :

灵敏性(sensitivity, SE):

$$
\mathrm{SE}=(\mathrm{TP} /(\mathrm{TP}+\mathrm{FN})) \times 100 \%
$$

特效性(specificity, SP):

$$
\mathrm{SP}=(\mathrm{TN} /(\mathrm{TN}+\mathrm{FP})) \times 100 \%
$$

总精度(accuracy, $Q$ ):

$$
Q=((\mathrm{TP}+\mathrm{TN}) /(\mathrm{TP}+\mathrm{TN}+\mathrm{FP}+\mathrm{FN})) \times 100 \%
$$

Matthews 相关系数 $(\mathrm{MCC})^{[67]}$.

$$
\mathrm{MCC}=\frac{\mathrm{TP} \times \mathrm{TN}-\mathrm{FN} \times \mathrm{FP}}{\sqrt{(\mathrm{TP}+\mathrm{FN})(\mathrm{TP}+\mathrm{FP})(\mathrm{TN}+\mathrm{FN})(\mathrm{TN}+\mathrm{FP})}}
$$

其中 TP 代表预测正确的正样本数, TN 代表预测正 确的负样本数, FP 代表预测错误的负样本数, FN 代 表预测错误的正样本数. 模型的敏感度分析 SE 用于 评估正样本的预测正确率, 特效性分析 SP 用于评估 负样本的预测正确率. $Q$ 用于评估总体样本的预测 正确率.

\section{2 结果和讨论}

\section{1 描述符笁选对 SVM 预测性能影响}

本研究中我们用 SVM 方法执行描述符变量的 筛选, 篮选结果列于表 1 . 最初每个训练集分子的描 述符数目为 1559 个, 经过预处理后, 描述符的数目 减少到 564 个, 说明在最初的 1559 个变量中存在大 量信息含量低或与其它描述符高度相关的描述符, 经过预处理可以去掉. 用这 564 个描述符通过五重 交叉验证方法建立 SVM 的分类预测模型, 正样本, 负样本和总样本的平均预测正确率分别是 $84.4 \%$, $72.0 \%$ 和 $79.6 \%, \mathrm{MCC}$ 为 0.573 . 进一步用 $F$-Score 值 递减排序笁选后, 描述符数量减少到 295 个, 通过五 重交叉验证建立SVM 模型, 正样本, 负样本和总样 本的平均预测正确率分别是 $87.9 \%, 70.5 \%$ 和 $81.2 \%, \mathrm{MCC}$ 为 0.602 . 对比两组数据可知, $F$-Score 排序步骤虽然没有明显提高模型的预测精度, 但大 大减少了描述符的数量, 有利于提高模型的计算效 率. 由于描述符的个数还很多, 故进一步用 Monte Carlo 模拟退火法进行笁选, 最终得到 37 个描述符, 此时 SVM 分类预测模型对正样本, 负样本和总样本 的平均预测正确率分别为 $92.7 \%, 81.0 \%$ 和 $88.2 \%$, $\mathrm{MCC}$ 为 0.749 . 由此可见, 模拟退火法在大量减少描 
表1＼cjkstart变量篮选对 SVM 预测精度的影响

Table 1 Effect of feature selection on the performance of SVM

\begin{tabular}{|c|c|c|c|c|c|c|c|c|c|c|}
\hline \multirow{2}{*}{ Step } & \multirow{2}{*}{ Optimal $\sigma$} & \multirow{2}{*}{ Cross-validation set } & \multicolumn{3}{|c|}{ Prediction for positive } & \multicolumn{3}{|c|}{ Prediction for negative } & \multirow{2}{*}{$Q(\%)$} & \multirow{2}{*}{$\mathrm{MCC}$} \\
\hline & & & $\mathrm{TP}$ & $\mathrm{FN}$ & SE (\%) & $\mathrm{TN}$ & $\mathrm{FP}$ & SP $(\%)$ & & \\
\hline \multirow[t]{7}{*}{ Step 1 (564) } & 12.0 & 1 & 55 & 8 & 87.3 & 26 & 14 & 65.0 & 78.6 & 0.542 \\
\hline & & 2 & 52 & 11 & 82.5 & 34 & 6 & 85.0 & 83.5 & 0.664 \\
\hline & & 3 & 55 & 8 & 87.3 & 27 & 13 & 67.5 & 79.6 & 0.564 \\
\hline & & 4 & 47 & 16 & 74.6 & 31 & 9 & 77.5 & 75.7 & 0.510 \\
\hline & & 5 & 57 & 6 & 90.5 & 26 & 14 & 65.0 & 80.6 & 0.584 \\
\hline & & average & & & 84.4 & & & 72.0 & 79.6 & 0.573 \\
\hline & & SD & & & 6.2 & & & 8.9 & 2.8 & 0.058 \\
\hline \multirow[t]{7}{*}{ Step 2 (295) } & 7.5 & 1 & 58 & 5 & 92.1 & 26 & 14 & 65.0 & 81.6 & 0.606 \\
\hline & & 2 & 53 & 10 & 84.1 & 33 & 7 & 82.5 & 83.5 & 0.659 \\
\hline & & 3 & 58 & 5 & 92.1 & 26 & 14 & 65.0 & 81.6 & 0.606 \\
\hline & & 4 & 51 & 12 & 81.0 & 31 & 9 & 77.5 & 79.6 & 0.578 \\
\hline & & 5 & 57 & 6 & 90.5 & 25 & 15 & 62.5 & 79.6 & 0.563 \\
\hline & & average & & & 87.9 & & & 70.5 & 81.2 & 0.602 \\
\hline & & SD & & & 5.1 & & & 8.9 & 1.6 & 0.037 \\
\hline \multirow[t]{7}{*}{ Step 3 (37) } & 4.0 & 1 & 59 & 4 & 93.7 & 32 & 8 & 80.0 & 88.4 & 0.753 \\
\hline & & 2 & 59 & 4 & 93.7 & 36 & 4 & 90.0 & 92.2 & 0.837 \\
\hline & & 3 & 59 & 4 & 93.7 & 32 & 8 & 80.0 & 88.4 & 0.753 \\
\hline & & 4 & 57 & 6 & 90.5 & 33 & 7 & 82.5 & 87.4 & 0.733 \\
\hline & & 5 & 58 & 5 & 92.1 & 29 & 11 & 72.5 & 84.5 & 0.669 \\
\hline & & average & & & 92.7 & & & 81.0 & 88.2 & 0.749 \\
\hline & & SD & & & 1.4 & & & 6.3 & 2.8 & 0.060 \\
\hline
\end{tabular}

Step 1: preprocessing, Step 2: ranking and backward selection, Step 3: Monte Carlo simulated annealing, the data in the parentheses are number of descriptors; $\sigma$ : exponent of the Gaussian kernel of SVM; SD: standard deviation; TP: truly predicted positive; FN: wrongly predicted positive; SE: sensitivity; TN: truly predicted negative; FP: wrongly predicted negative; SP: specificity; $Q$ : overall prediction accuracy;

MCC: Matthews correlation coefficient

述符数量的同时能够大大提高模型的预测精度, 说 明干扰变量和噪音变量已经被排除, 得到了与分子 活性相关性很强的描述符. 最初的 1559 个描述符及 篮选出的 37 个描述符列于 Supporting Information 中的表 $\mathrm{S} 1$ 和 $\mathrm{S} 2$.

\section{$2.2 S V M$ 模型 $y$-scrambling 验证}

为了评估所建模型对偶然相关的依赖程度, 本 文采用 $y$-scrambling 方法对 SVM 所建的模型进行验 证. 首先将训练集样本中的输入变量 $x$ 保持不变, 随 机将部分样本对应的分类标号 $y$ 改变类别, 使这些 样本的输入变量和类别号不再对应, 以消除两者之 间存在的内在定量关系; 随后, 针对上述改变类别 的训练集, 采用本文的混合描述符篮选方法结合 SVM 通过五重交叉验证建立新的预测模型; 重复上 述过程 30 次, 并将其预测结果与基于原始样本数据 建立的实际预测模型进行比较, 其预测结果见 Supporting Information 中的表 S3.

从附表 $\mathrm{S} 3$ 可以看出, 通过 $y$-scrambling 方法验 证 SVM 模型, 正样本, 负样本和总样本的平均预测
精度分别在 $72.7 \%-82.5 \%, 41.0 \%-53.0 \%$ 和 $62.1 \%$ $69.1 \%$, 很明显, 预测精度远低于基于原始样本数据 建立的实际预测模型. 这说明我们构建的 SVM 模型 不存在偶然相关性.

\section{3 其他机器学习方法}

同时, 为了检验所选描述符能否真正区别开不 同类别的样本, 我们把 SVM 方法篮选出的 37 个描 述符直接用于建立 ANN 和 $k-\mathrm{NN}$ 的分类模型, 结果 见表 2. 从表中可知, 机器学习方法对正样本、负样 本、总样本的平均预测精度和 MCC 分别在 $87.3 \%-92.7 \% 、 67.0 \%-81.0 \% 、 79.4 \%-88.2 \%$ 和 $0.561-$ $0.749, \mathrm{SVM}$ 模型给出了最好的预测结果: $\mathrm{SE}=$ $92.7 \%, \mathrm{SP}=81.0 \%, Q=88.2 \%, \mathrm{MCC}=0.749$. 上面的结 果表明我们所选择的描述符也能用于其它机器学 习方法建立分类模型并且可以得到良好的预测结 果, 所选的描述符是与乙酰胆碱酯酶抑制剂活性真 正相关的.

\section{4 外部独立测试集}

把所有的训练集化合物用篮选出来的最优描 
述符子集和最优参数建立模型, 然后用外部独立测 试集来评价模型. 在讨论外部测试之前, 先讨论模 型的应用域.

机器学习方法所建立的模型是基于特定的训 练集而产生的, 所以必定有其应用的范围, 只有确 定模型的应用范围, 我们才知道所建模型对未知事 物预测的可信度. 模型的应用域就是用来讨论模型 应用范围的. 本论文中关于应用域的研究主要采用 描述符空间(即训练集化合物所覆盖的描述符空间 的组合, 也称之为描述符域 $\left.{ }^{[8]}\right)$ 的方法, 从建立模型 所使用的训练集和描述符出发进行研究. 本文根据 欧氏距离的方法来表征描述符域, Tropsha等 ${ }^{[69]}$ 定义 了应用域距离的阀值 $D_{\mathrm{T}}$ :

$$
D_{\mathrm{T}}=\langle D\rangle+Z s
$$

其中 $Z$ 为阈值参数, $\langle D\rangle$ 和 $s$ 是训练集中所有化合物 间欧氏距离平均值和标准差. 我们根据类似的方法 定义应用域 $S_{\mathrm{T}}$ 为:

$$
S_{\mathrm{T}}=\langle S\rangle-Z \sigma
$$

$\langle S\rangle$ 和 $\sigma$ 是训练集中所有化合物的归一化 Tanimoto 相似性系数和标准差, $Z$ 为任意的阈值参数, 目的是 调控得到最大的应用域, 我们设置 $Z$ 缺省值为 0.5 . 如果用来测试的化合物的 Tanimoto 相似性系数低 于 $S_{\mathrm{T}}$, 则该化合物被认为是超出了应用领域, 此时预
测是不可靠的.

把篮选后 37 个描述符用于训练集的计算, 然后 对所得的分子描述符数据进行标准化处理, 即对每 个变量先减去样本平均值, 再除以样本方差, 从而 消除不同量纲对变量的影响, 然后通过主成分分析 (principal component analysis, PCA)消除多余的信 息, 并选取信息含量的加和大于 $90 \%$ 的主成分 (17 个)用于样本间相似性系数的计算. 对于训练集, 经 过归一化的 Tanimoto 系数平均值为 0.778 , 标准偏差 为 0.106 , 由式(19)计算出 $S_{\mathrm{T}}$ 为 0.725 . 对于 172 个独 立的测试集分子, 严格地说只有 9 个化合物没有 在应用域范围内, 它们距离训练集样本最近分子的 Tanimoto 系数值分别为: $0.656,0.682,0.720,0.680$, $0.661,0.696,0.687,0.634$ 和 0.711 . 尽管这些 Tanimoto 系数低于 $S_{\mathrm{T}}$ 值 $(0.725)$, 但是彼此很接近, 所以我们 认为这 9 个分子也在所建模型的应用域范围内.

表 3 列出了模型对独立测试集的预测结果, 可 以看到正样本, 负样本, 总样本的预测精度和 $\mathrm{MCC}$ 分别在 $93.3 \%-100.0 \%, 74.6 \%-89.6 \%, 86.1 \%-95.9 \%$ 和 0.704-0.916. 结果表明机器学习方法所建模型对 外部独立测试分子取得了良好的预测结果, 所建模 型不存在过拟合的现象, 同时也可以看出基于 SVM 方法所建模型的预测精度要明显好于基于 $k-\mathrm{NN}$ 和

表 2 五重交叉验证用于 3 种机器学习方法预测结果的比较

\begin{tabular}{|c|c|c|c|c|c|c|c|c|c|c|}
\hline \multirow{2}{*}{ Method } & \multirow{2}{*}{ Parameter } & \multirow{2}{*}{ 5-cross validation } & \multicolumn{3}{|c|}{ Prediction for positive } & \multicolumn{3}{|c|}{ Prediction for negative } & \multirow{2}{*}{$Q(\%)$} & \multirow{2}{*}{$\mathrm{MCC}$} \\
\hline & & & $\mathrm{TP}$ & $\mathrm{FN}$ & SE (\%) & $\mathrm{TN}$ & $\mathrm{FP}$ & SP (\%) & & \\
\hline \multirow[t]{6}{*}{ ANN } & $n^{\mathrm{a}}=20$ & 1 & 57 & 6 & 90.5 & 23 & 17 & 57.5 & 77.7 & 0.520 \\
\hline & & 2 & 54 & 9 & 85.7 & 29 & 11 & 72.5 & 80.6 & 0.588 \\
\hline & & 3 & 57 & 6 & 90.5 & 24 & 16 & 60.0 & 78.6 & 0.542 \\
\hline & & 4 & 53 & 10 & 84.1 & 32 & 8 & 80.0 & 82.5 & 0.636 \\
\hline & & 5 & 54 & 9 & 85.7 & 26 & 14 & 65.0 & 77.7 & 0.522 \\
\hline & & average & & & 87.3 & & & 67.0 & 79.4 & 0.561 \\
\hline \multirow[t]{6}{*}{$\mathrm{k}-\mathrm{NN}$} & $k^{\mathrm{b}}=3$ & 1 & 57 & 6 & 90.5 & 26 & 14 & 65.0 & 80.6 & 0.584 \\
\hline & & 2 & 57 & 6 & 90.5 & 32 & 8 & 80.0 & 86.4 & 0.712 \\
\hline & & 3 & 59 & 4 & 93.7 & 26 & 14 & 65.0 & 82.5 & 0.629 \\
\hline & & 4 & 53 & 10 & 84.1 & 26 & 14 & 65.0 & 76.7 & 0.502 \\
\hline & & 5 & 55 & 8 & 87.3 & 24 & 16 & 60.0 & 76.7 & 0.498 \\
\hline & & average & & & 89.2 & & & 67.0 & 80.6 & 0.585 \\
\hline \multirow[t]{6}{*}{ SVM } & $\sigma=4.0$ & 1 & 59 & 4 & 93.7 & 32 & 8 & 80.0 & 88.4 & 0.753 \\
\hline & & 2 & 59 & 4 & 93.7 & 36 & 4 & 90.0 & 92.2 & 0.837 \\
\hline & & 3 & 59 & 4 & 93.7 & 32 & 8 & 80.0 & 88.4 & 0.753 \\
\hline & & 4 & 57 & 6 & 90.5 & 33 & 7 & 82.5 & 87.4 & 0.733 \\
\hline & & 5 & 58 & 5 & 92.1 & 29 & 11 & 72.5 & 84.5 & 0.669 \\
\hline & & average & & & 92.7 & & & 81.0 & 88.2 & 0.749 \\
\hline
\end{tabular}

Table 2 Comparison of the prediction results from three machine learning methods by using 5-fold cross validation 
表 33 种机器学习方法对外部独立测试集预测结果的比较

Table 3 Comparing predicted results based on three machine learning methods using independent validation sets

\begin{tabular}{cccccccccc}
\hline \multirow{2}{*}{ Method } & \multirow{2}{*}{ Parameter } & \multicolumn{7}{c}{ External validation method } \\
\cline { 2 - 10 } & & TP & FN & SE (\%) & TN & FP & SP (\%) & $Q(\%)$ & MCC \\
\hline ANN & $n=20$ & 98 & 7 & 93.3 & 50 & 17 & 74.6 & 86.1 & 0.704 \\
$k$-NN & $k=3$ & 99 & 6 & 94.3 & 56 & 11 & 83.6 & 90.1 & 0.791 \\
SVM & $\sigma=4.0$ & 105 & 0 & 100.0 & 60 & 7 & 89.6 & 95.9 & 0.916 \\
\hline
\end{tabular}

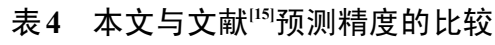

Table 4 Comparison of the prediction accuracies of our models with literature ${ }^{[15]}$

\begin{tabular}{|c|c|c|c|c|c|c|c|c|c|}
\hline \multirow{2}{*}{ Study } & \multirow{2}{*}{ Method } & \multicolumn{4}{|c|}{ Training set accuracy } & \multicolumn{4}{|c|}{ External test set accuracy } \\
\hline & & Sample number & SE $(\%)$ & SP (\%) & $Q(\%)$ & Sample number & SE (\%) & SP (\%) & $Q(\%)$ \\
\hline \multirow[t]{3}{*}{ Lv et al. ${ }^{[15]}$} & C4.5 DT & & & & & 116 & 85.2 & 62.9 & 78.4 \\
\hline & $k$-NN & & & & & 116 & 87.7 & 74.3 & 83.6 \\
\hline & SVM-RFE ${ }^{a}$ & 447 & 88.0 & 76.4 & 84.0 & 116 & 88.9 & 82.9 & 87.1 \\
\hline \multirow[t]{3}{*}{ this study } & ANN & 515 & 87.3 & 67.0 & 79.4 & 172 & 93.3 & 74.6 & 86.1 \\
\hline & $k$-NN & 515 & 89.2 & 67.0 & 80.6 & 172 & 94.3 & 83.6 & 90.1 \\
\hline & SVM-MC & 515 & 92.7 & 81.0 & 88.2 & 172 & 100.0 & 89.6 & 95.9 \\
\hline
\end{tabular}

${ }^{a} \mathrm{SVM}$ with RFE for feature selection, ${ }^{\mathrm{b}} \mathrm{SVM}$ with Monte Carlo simulated annealing for feature selection

ANN建立的模型.

\section{5 文献结果比较}

将我们建立的模型的预测结果与文献[15]预测 的结果进行比较, 以便检验我们模型的预测水平. 但是需要指出的是直接和不同模型的预测结果相 比较不太合理, 因为不同的模型采用了不同的机器 学习方法、不同的训练集和测试集样本、不同的分 子描述符和不同的验证方法. 但是可以进行尝试性 的比较, 以便对乙酰胆碱酯酶抑制剂的预测精度进 行大概的评估. 本研究主要与 $\mathrm{Lv}$ 等 ${ }^{[15}$ 建立的模型进 行比较, 他们也使用了三种机器学习方法建立了分 类模型并取得了良好的预测结果. 比较结果列于表 4 , 从表中可以看出, 本文的机器学习方法所建模型 的预测精度要接近或者好于文献报道的结果, 尤其 是 SVM-MC 模型, 无论是交叉验证还是外部独立测 试的结果, 都明显好于文献报道结果, 而且我们所 用样本的数量更多, 化合物结构更具有多样性, 应 用域覆盖了更大的化学空间. 所以我们建立的模型 尤其是 SVM-MC 模型更加适用于对未知的乙酰胆 碱酯酶抑制剂进行预测.

\section{3 结 论}

本文对结构多样性较大的乙酰胆碱酯酶抑制 剂化合物数据集计算了 1559 个分子描述符, 然后通 过一套混合描述符篎选方法篎选出和抑制剂活性 相关性很强的 37 个描述符, 最后用 SVM, $k-\mathrm{NN}$ 和 ANN 建立乙酰胆碱酯酶抑制剂的分类预测模型. 研
究结果表明: 变量耖选在大大减少描述符个数的同 时能大大提高模型的预测能力; 本文所建的 SVM$\mathrm{MC}$ 模型要优于其他机器学习方法建立的模型, 且 预测精度明显好于文献已报道的结果, 从而可以用 于计算机辅助笁选具有乙酰胆碱酯酶抑制活性的 先导化合物.

Supporting Information Available: Table S1 listed 1559 molecular descriptors. The selected molecular descriptors were listed in Table S2. Table S3 listed the results of $y$-scrambling test on SVM model. This information is available free of charge via the internet at http://www.whxb.pku.edu.cn.

\section{References}

1 Dekosky, S. T.; Scheff, S. W. Ann. Neurol., 1990, 27: 457

2 Terry, R. D.; Masliah, E.; Salmon, D. P.; Butters, N.; DeTeresa, R.; Hill, R.; Hansen, L. A.; Katzman, R. Ann. Neurol., 1991, 30: 572

3 Rösler, M.; Anand, R.; Cicin-Sain, A.; Gauthier, S.; Agid, Y.; DalBianco, P.; Stähelin, H. B.; Hartman, R.; Gharabawi, M. Br. Med. J., 1999, 318: 633

4 Whitehouse, P. J. Acta Neurologica Scandinavica Supplement, 1993, 149: 42

5 Kelly, C. A.; Harvey, R. J.; Cayton, H. Br. Med. J., 1997, 314: 693

6 Scott, L. J.; Goa, L. K. Drugs, 2000, 60: 1095

7 Gottwald, M. D.; Rozanski, R. I. Expert Opinion on Investigational Drugs, 1999, 8: 1673

8 Hasegawa, K. J. Chem. Inf. Comput. Sci., 1999, 39: 112

9 Recanatini, M.; Cavalli, A.; Belluti, F.; Piazzi, L.; Rampa, A.; Bisi, A.; Gobbi, S.; Valenti, P.; Andrisano, V.; Bartolini, M.; Cavrini, V. J. Med. Chem., 2000, 43: 2007 
10 Sheng, R.; Shen, Y. H.; Lin, X.; Luo, Y.; Fan, Y. J.; Li, J. Y.; Xia, H. R.; Hu, Y. Z. Chinese Journal of Medicinal Chemistry, 2007, 17: 348 [盛 荣, 申艳红, 林 肖, 罗 蕴, 范永剑, 李静雅, 夏 海蓉, 胡永洲. 中国药物化学杂志, 2007, 17: 348]

11 Liu, A. L.; Guang, H. M.; Zhu, L. Y.; Du, G. H.; Li, M. Y.; Wang, Y. T. Sci. China Ser. C-Life Sci., 2007, 37: 503 [刘艾林, 光红 梅, 朱莉亚, 杜冠华, 李铭源, 王一涛. 中国科学 C辑: 生命科学, 2007, 37: 503]

12 Mizutani, M. Y.; Itai, A. J. Med. Chem., 2004, 47: 4818

13 Jiang, Y. R.; Xu, H.; Chen, F. J.; Ma, G. J. Acta Phys. -Chim. Sin., 2009, 25: 1379 [蒋玉仁, 许 慧, 陈芳军, 马贯军. 物理化学学 报, 2009, 25: 1379]

14 Zheng, Q. C.; Chu, H. Y.; Niu, R. J.; Sun, C. C. Sci. China Ser. BChem., 2009, 39: 1454 [郑清川, 楚慧郢, 牛瑞娟, 孙家锤. 中国 科学 B 辑: 化学, 2009, 39: 1454]

15 Lv, W.; Xue, Y. European Journal of Medicinal Chemistry, 2010, 45: 1167

16 Czerminski, R.; Yasri, A.; Hartsourgh, D. Quant. Struct. -Act. Relat., 2001, 20: 227

17 Tetko, I. V.; Luik, A. I.; Poda, G. I. J. Med. Chem., 1993, 36: 811

18 Ruiz, V. E. Pattern Recognition Letters, 1986, 4: 145

19 Ishihara, Y.; Hirai, K.; Miyamoto, M.; Goto, G. J. Med. Chem., 1994, 37: 2292

20 Villalobos, A.; Blake, J. F.; Bigger, C. K.; Butler, T. W.; Chapin, D. S.; Chen, Y. L.; Ives, J. L.; Jones, S. B.; Liston, D. R.; Nagel, A. A.; Nason, D. M.; Nielsen, J. A.; Shalaby, I. A.; White, W. F. J. Med. Chem., 1994, 37: 2721

21 Chen, Y. L.; Nielsen, J.; Hedberg, K.; Dunaiskis, A.; Jones, S.; Russo, L.; Johnson, J.; Ives, J.; Liston, D. J. Med. Chem., 1992, 5 1429

22 Sugimoto, H.; Tsuchiya, Y.; Sugumi, H.; Higurashi, K.; Karibe, N.; Iimura, Y.; Sasaki, A.; Araki, S.; Yamanishi, Y.; Yamatsu, K. J. Med. Chem., 1992, 35: 4542

23 Villalobos, A.; Butler, T. W.; Chapin, D. S.; Chen, Y. L.; DeMattos, S. B.; Ives, J. L.; Jones, S. B.; Liston, D. R.; Nagel, A. A.; Nason, D. M.; Nielsen, J. A.; Ramirez, A. D,; Shalaby, I. A.; White, W. F. J. Med. Chem., 1995, 38: 2802

24 Rampa, A.; Bisi, A.; Valenti, P.; Recanatini, M.; Cavalli, A.; Andrisano, V.; Cavrini, V.; Fin, L.; Buriani, A.; Giusti, P. J. Med. Chem., 1998, 41: 3976

25 Contreras, J. M.; Rival, Y. M.; Chayer, S.; Bourguignon, J. J.; Wermuth, C. G. J. Med. Chem., 1999, 42: 730

26 Nagel, A. A.; Liston, D. R.; Jung, S.; Mahar, M.; Vincent, L. A.; Chapin, D.; Chen, Y. L.; Hubbard, S.; Ives, J. L.; Jones, S. B. Nielsen, J. A.; Ramirez, A.; Shalaby, I. A.; Villalobos, A.; White, W. F. J. Med. Chem., 1995, 38: 1084

27 Vidaluc, J. L.; Calmel, F.; Bigg, D. C. H.; Carilla, E.; Briley, M. J. Med. Chem., 1995, 38: 2969

28 Carlier, P. R.; Chow, E. S. H.; Han, Y.; Liu, J.; Yazal, J. E.; Pang, Y. P. J. Med. Chem., 1999, 42: 4225

29 Kenna, M. T. M.; Proctor, G. R.; Young, L. C.; Harvey, A. L.
J. Med. Chem., 1997, 40: 3516

30 Sugimoto, H.; Iimura, Y.; Yamanishi, Y.; Yamatsu, K. J. Med Chem., 1995, 38: 4821

31 Rampa, A.; Piazzi, L.; Belluti, F.; Gobbi, S.; Bisi, A.; Bartolini, M.; Andrisano, V.; Cavrini, V.; Cavalli, A.; Recanatini, M.; Valenti, P. J. Med. Chem., 2001, 44: 3810

32 Contreras, J. M.; Parrot, I.; Sippl, W.; Rival, Y. M.; Wermuth, C. G. J. Med. Chem., 2001, 44: 2707

33 Hu, M. K.; Wu, L. J.; Hsiao, G.; Yen, M. H. J. Med. Chem., 2002, 45: 2277

34 Recanatini, M.; Cavalli, A.; Belluti, F.; Piazzi, L.; Rampa, A.; Bisi, A.; Gobbi, S.; Valenti, P.; Andrisano, V.; Bartolini, M.; Cavrini, V. J. Med. Chem., 2000, 43: 2007

35 Belluti, F.; Rampa, A.; Piazzi, L.; Bisi, A.; Gobbi, S.; Bartolini, M.; Andrisano, V.; Cavalli, A.; Recanatini, M.; Valenti, P. J. Med. Chem., 2005, 48: 4444

36 Pilar, M. R.; Rubio, L.; Esther, G. P.; Dorronsoro, I.; Maria, M. M.; Valenzuela, R.; Usan, P.; Austria, C.; Bartolini, M.; Andrisano, V.; Axel, B. C.; Orozco, M.; Luque, F. J.; Medina, M.; Martinez, A. J. Med. Chem., 2005, 48: 7223

37 Sauvaitre, T.; Barlier, M.; Herlem, D.; Gresh, N.; Chiaroni, A.; Guenard, D.; Guillou, C. J. Med. Chem., 2007, 50: 5311

38 Cardoso, C. L.; Ian, C. G.; Silva, D. H. S.; Furlan, M.; Epifanio, R. A.; Pinto, A. C.; Rezende, C. M.; Lima, J. A.; Bolzani, V. S. J. Nat. Prod., 2004, 67: 1882

39 Bolognesi, M. L.; Cavalli, A.; Valgimigli, L.; Bartolini, M.; Rosini, M.; Andrisano, V.; Recanatini, M.; Melchiorre, C. J. Med. Chem., 2007, 50: 6446

40 Kavitha, C. V.; Gaonkar, S. L.; Chandra, J. N.; Sadashiva, C. T.; Rangappa, K. S. Bioorganic \& Medicinal Chemistry, 2007, 15: 7391

41 Bianchi, D. A.; Hirschmann, G. S.; Theoduloz, C.; Bracca, A. B. J.; Kaufman, T. S. Bioorganic \& Medicinal Chemistry Letters, 2005, 15: 2711

42 Sheng, R.; Lin, X.; Li, J. Y; Jiang, Y. K.; Shang, Z. C.; Hu, Y. Z. Bioorganic \& Medicinal Chemistry Letters, 2005, 15: 3834

43 Ishichi, Y.; Sasaki, M.; Setoh, M.; Tsukamoto, T.; Miwatashi, S.; Nagabukuro, H.; Okanishi, S.; Imai, S.; Saikawa, R.; Doi, T.; Ishihara, Y. Bioorganic \& Medicinal Chemistry Letters, 2005, 13: 1901

44 Wang, B.; Mai, Y. C.; Li, Y.; Hou, J. Q.; Huang, S. L.; Ou, T. M.; Tan, J. H.; An, L. K.; Li, D.; Gu, L. Q.; Huang, Z. S. European J. Med. Chem., 2010, 45: 1415

45 Martinez, A.; Fernandez, E.; Castro, A.; Conde, S.; Isabel, R. F.; Banos, J. E.; Badia, A. J. Med. Chem., 2000, 35: 913

46 Shao, D.; Zou, C. Y.; Luo, C.; Tang, X. C.; Li, Y. C. Bioorganic \& Medicinal Chemistry Letters, 2004, 14: 4639

47 Belluti, F.; Piazzi, L.; Bisi, A.; Gobbi, S.; Bartolini, M.; Cavalli, A.; Valenti, P.; Rampa, A. European Journal of Medicinal Chemistry, 2009, 44: 1341

48 Rampa, A.; Bisi, A.; Belluti, F.; Gobbi, S.; Valenti, P.; Andrisano, 
V.; Cavrini, V.; Cavalli, A.; Recanatini, M. Bioorganic \& Medicinal Chemistry, 2000, 8: 497

49 Toda, N.; Tago, K.; Marumoto, S.; Takami, K.; Ori, M.; Yamada, N.; Koyama, K.; Naruto, S.; Abe, K.; Yamazaki, R.; Hara, T.; Aoyagi, A.; Abe, Y.; Kaneko, T.; Kogen, H. Bioorganic \& Medicinal Chemistry, 2003, 11: 1935

50 Zaheer, U. H.; Mahmood, U.; Jehangir, B. Chem. Biol. Drug. Des., 2009, 74: 571

51 Pan, L.; Tan, J. H.; Hou, J. Q.; Huang, S. L.; Gu, L. Q.; Huang, Z. S. Bioorganic \& Medicinal Chemistry Letters, 2008, 18: 3790

52 Marco, J. L.; Rios, C.; Garcia, A. G.; Villarroya, M.; Carreiras, M. C.; Martins, C.; Eleuterio, A.; Morreale, A.; Orozco, M.; Luque, F. J. Bioorganic \& Medicinal Chemistry, 2004, 12: 2199

53 Shen, Q.; Peng, Q.; Shao, J. L.; Liu, X. F.; Huang, Z. S.; Pu, X. Z.; Ma, L.; Li, Y. M.; Chan, A. S. C.; Gu, L. Q. European Journal of Medicinal Chemistry, 2005, 40: 1307

54 Martin, L. L.; Davis, L.; Klein, J. T.; Nemoto, P.; Olsen, G. E.; Bores, G. M.; Camacho, F.; Petko, W. W.; Rush, D. K.; Selk, D.; Smith, C. P.; Vargas, H. M.; Winslow, J. T.; Effland, R. C.; Fink, D. M. Bioorganic \& Medicinal Chemistry Letters, 1997, 7: 157

55 Sugimoto, H.; Yamanishi, Y.; Iimura, Y.; Kawakami, Y. Current Medicinal Chemistry, 2000, 7: 303

56 Stewart, J. J. P. J. Comput. Chem., 1989, 10: 221

57 Willett, P.; Barnard, J. M.; Downs, G. M. J. Chem. Inf. Comput.

\section{Sci., 1998, 38: 983}

58 Li, Z. R.; Han, L. Y.; Xue, Y.; Yap, C. W.; Li, H.; Jiang, L.; Chen, Y. Z. Biotechnol. Bioeng., 2007, 97: 389

59 Yang, C. W.; Su, J. Y.; Tsou, A. P.; Chau, G. Y.; Liu, H. L.; Chen, C. H.; Chien, C. Y.; Chou, C. K. Biochem. Biophys. Res. Commun., 2005, 330: 489

60 Fisher, R. A. Annals of Eugenics, 1936, 7: 179

61 Ajmani, S.; Jadhav, K.; Kulkarni, S. A. J. Chem. Inf. Model., 2006, 46: 24

62 Li, P.; Tan, N. X.; Rao, H. B.; Li, Z. R.; Chen, Y. Z. Acta Phys. Chim. Sin., 2009, 25: 1581 [李 平, 谈宁馨, 饶含兵, 李泽荣, 陈宇综. 物理化学学报, 2009, 25: 1581]

63 Kennard, R. W.; Stone, L. A. Technometrics, 1969, 11: 137

64 Liu, H.; Papa, E.; Gramatica, P. Chem. Res. Toxicol., 2006, 19: 1540

65 Liu, H.; Gramatica, P. Bioorganic \& Medicinal Chemistry, 2007, 15: 5251

66 Bhasin, M.; Zhang, H.; Reinherz, E. L.; Reche, P. A. FEBS. Lett., 2005, 579: 4302

67 Matthews, B. W. Biochim. Biophys. Acta, 1975, 405: 442

68 Dimitrov, S.; Dimitrova, G.; Pavlov, T.; Dimitrova, N.; Patlewicz, G.; Niemela, J.; Mekenyan, O. J. Chem. Inf. Model., 2005, 45: 839

69 Tropsha, A.; Golbraikh, A. Curr. Pharm. Des., 2007, 13: 3494 